\title{
Efficient and reusable iron-zinc oxide catalyst for oxidative desulfurization of model fuel
}

\begin{abstract}
In this study, $\mathrm{Fe}-\mathrm{ZnO}$ catalyst synthesized via modified sol-gel technique with different $\mathrm{Fe}$ doping ratio $(2,3$, and 5$) \mathrm{wt} \%$ was explored for oxidative desulfurization (ODS) of model fuel. The sol-gel technique was adopted without the use of surfactants. The catalysts were characterized by several means of characterization techniques (TGA, XRD, FTIR, N2physisorption, XPS, FESEM-EDX and NH3-TPD). The characterization results clearly showed that sol-gel technique is a suitable method to synthesize highly crystalline metal oxide materials with smaller particle size, higher surface area and tunable acidic properties. The ODS reaction conditions and Fe metal loading were found to influence the dibenzothiophene (DBT) removal efficiency. The catalytic ODS data showed that Fe-ZnO with $5 \mathrm{wt} \%$ of Fe catalyst is capable in total removal of DBT within shorter reaction time under mild reaction conditions in the presence of $\mathrm{H} 2 \mathrm{O} 2$ as an oxidant. The catalyst is reusable for six consecutive cycles of reaction without regeneration steps and the characteristic of spent catalyst was confirmed with XRD and FTIR analysis. The close catalytic cycle involving $\mathrm{H} 2 \mathrm{O} 2$ as an oxidant was shown through proposed mechanistic pathway.
\end{abstract}

Keyword: Oxidative desulfurization; Fe-ZnO; Sol-gel; Fuel; Hydrogen peroxide 